\title{
Characteristics of Quinolone Resistance in Multidrug-Resistant Acinetobacter baumannii Strains Isolated from General Hospitals
}

\author{
Choong Ki Hong (iD ${ }^{1}$, Joon Kim (iD) ${ }^{2}$ and Ga-Yeon Kim (DiD ${ }^{3, *}$ \\ ${ }^{1}$ Department of Clinical Laboratory Science, Graduate School of Dankook University, Chungnam, South Korea \\ ${ }^{2}$ Department of Laboratory Medicine, Ajou University Hospital, Suwon, South Korea \\ ${ }^{3}$ Department of Public Health, Dankook University Graduate School, Chungnam, South Korea \\ "Corresponding author: Department of Public Health, Dankook University Graduate School, Chungnam, South Korea. Tel: +82-415501493, Fax: +82-415501490, Email: \\ sysnhj77@gmail.com
}

Received 2021 April 14; Revised 2021 May 21; Accepted 2021 May 22.

\begin{abstract}
Background: Acinetobacter baumannii is the causative agent in various types of hospital-acquired infections, including respiratory, urinary tract, and wound infections.

Objectives: This study investigated the primary mechanisms underlying quinolone resistance in A. baumannii strains, isolated from samples collected from general hospitals.

Methods: Ninety-eight strains of A. baumannii were isolated from clinical specimens from general hospitals from 2017 - 2019. Antimicrobial susceptibility, efflux pump inhibition tests, multilocus sequence typing (MLST), and matrix-assisted laser desorption/ionization time-of-flight mass spectrometry analyses were conducted on 64 strains, and the $b l a_{\text {oxa-51-like gene sequence was }}$ detected.

Results: In the antimicrobial susceptibility test, 78.1\% $(n=50)$ of the strains exhibited resistance to ciprofloxacin, a quinolone antibiotic, and 57.8\% ( $\mathrm{n}=37)$ strains were multidrug resistant (MDR). For 18 strains, the minimum inhibitory concentration of ciprofloxacin reduced in presence of an efflux pump inhibitor. Sequence analysis revealed that in 50 strains of A. baumannii, the codon for serine (TCA) in gyrA was replaced by that for leucine (TTA), whereas in 43 strains, the codon for serine (TCG) in parC was replaced by that for leucine (TTG). Multilocus sequence typing analysis confirmed 18 sequence types, and allelic number analysis showed the presence of nine gyrB alleles, with gyrB3 showing the highest frequency (62.5\%).

Conclusions: The findings of this study will be useful in improving treatment efficiency and preventing the spread of $A$. baumannii (both MDR and non-MDR strains).
\end{abstract}

Keywords: Multilocus Sequence Typing, Drug Resistance, Multiple, Spectrometry, Mass, Matrix-assisted Laser Desorption-ionization.

\section{Background}

Acinetobacter baumannii is an aerobic Gram-negative bacillus. This major pathogen causes various types of hospital-acquired infections, such as respiratory, urinary tract, and wound infections (1). Acinetobacter baumannii is resistant to major antibiotics, including $\beta$ lactams, aminoglycosides, and quinolones. Outbreaks of multidrug-resistant (MDR) bacteria significantly affect the treatment of patients with severe infections (2). Ciprofloxacin, levofloxacin, and sparfloxacin are representative quinolone antibiotics, which exhibit antimicrobial activity against Gram-positive and Gram-negative bacteria and have shown clinical efficacy in treating respiratory infections. They can be orally administered due to their stable chemical structures and excellent tissue permeability. Quinolones are known to exhibit excellent permeability as they usually enter the cells via porin (membrane transport protein) located in the outer membrane of bacterial cells (3). In bacteria, efflux pumps actively transport antibiotics from cells and are known to be the primary cellular components mediating the natural resistance of Gram-negative bacteria to various antibiotics (4).

In recent times, pulsed-field gel electrophoresis and multilocus sequence typing (MLST) have been used to trace the lineage or origin of most epidemic strains, including those of $A$. baumannii. Among the structural genes with varying sequences in different species, primers for gltA, gyrB, gdhB, recA, cpn60, gpi, rpoD, pgm, quiA, and pcaf are used for $A$. baumannii. Among these, the high allelic number of gyrB indicates frequent mutations, which also leads to the expression of various STs $(5,6)$. Multiplex PCR analysis using the gyrB primer was confirmed to be 
the most rapid method for distinguishing and identifying A. baumannii and four other A. baumannii complexes $(5,6)$. Matrix-assisted laser desorption/ionization-time of flight(MALDI-TOF) mass spectrometry (MS) analysis identifies the measurable molecular weight (100 Da to $100 \mathrm{kDa}$ ) and the bacterial cell count corresponding to one or two colonies (105 to 106) by examining the protein peaks (7).

\section{Objectives}

The effluent pump inhibition influence test and sequencing of quinolone resistance-determining region (QRDR) of gyrA and parC genes were conducted to determine the cause of resistance of quinolone-based antibacterial agents. In addition, MLST was performed to determine the genetic correlation of the structural gene gyrB with the amino acid variation at a specific site of gyrA called QRDR. The protein molecular weight of each strain was measured and compared with the results of antimicrobial sensitivity tests.

\section{Methods}

\subsection{Strain Isolation}

This study was approved by the Institutional Review Board (IRB-202010027) of Dankook University. Ninetyeight strains of $A$. baumannii isolated from sputum, wound debris, urine, and pus samples obtained from the Gyeonggi Southern General Hospital from 2017 - 2019 were identified using the VITEK® 2 XL (bioMérieux, Marcyl'Etoile, France) GN-card. The detection primers used in PCR included OXA-51-like F: 5'-TAA TGC TTT GAT CGG CCT TG-3' and OXA-51-like R: 5'-TGG ATT GCA CTT CAT CTT GG-3'. The PCR product of $353 \mathrm{bp}$ was confirmed at $302 \mathrm{~nm}$ using the Molecular Imager Gel DOCTM XR+ System (Bio-Rad, USA) (8).

\subsection{Antimicrobial Susceptibility Test}

Antimicrobial susceptibility was assessed using the VITEK® 2 XL AST-N225 card for measuring the minimum inhibitory concentration (MIC). The following 17 antibiotics were used: penicillin with extended spectrum (piperacillin), $\beta$-lactam $/ \beta$-lactamase inhibitor combinations (ampicillin/sulbactam, piperacillin/tazobactam, and ticarcillin/clavulanic acid), cephems (cefotaxime, ceftazidime, and cefepime), carbapenems (imipenem and meropenem), aminoglycosides (amikacin and gentamicin), folate pathway inhibitors (trimethoprim/sulfamethoxazole), quinolone (levofloxacin and ciprofloxacin), tetracyclines (minocycline), glycylcyclines (tigecycline), polymyxin (colistin), and monobactam (aztreonam). The antimicrobial susceptibility test was performed in compliance with the Clinical and Laboratory
Standards Institute (CLSI) guidelines (2020, M100-S30) (9). Escherichia coli ATCC 25922 and Pseudomonas aeruginosa ATCC 27853 were used as standard strains.

\subsection{Efflux Pump Inhibition Test}

The efflux pump inhibition test was performed using phenylalanine-arginine $\beta$-naphthylamide $(\mathrm{PA} \beta \mathrm{N})$, an efflux pump inhibitor (EPI), on 50 strains with confirmed ciprofloxacin resistance in the antimicrobial susceptibility test. The second- and third-generation quinolone antibiotics, ciprofloxacin and levofloxacin (Sigma-Aldrich, USA) respectively, were used. $\mathrm{Pa} \beta \mathrm{N}$ was administered at 100 $\mu \mathrm{g} / \mathrm{mL}$, and comparison of MIC reduction of the antibiotic in presence and absence of EPI was measured (10). The MIC of the antibiotic was measured using the solid medium dilution method, as directed in the CLSI guidelines (2020, M100-S30) (9). E. coli ATCC 25922 was used as the standard strain.

\subsection{Analysis of $Q R D R$ Sequence of gyrA and parC}

Sequence analysis was performed on 50 ciprofloxacinresistant strains of $A$. baumannii to determine the presence of mutation in each gene (11). The analyzed nucleotide sequences were the standard A. baumannii gyrA and parC sequences. The gyrA and parC sequences of the strains were compared to the corresponding standard gene sequences (GenBank accession numbers X82165 and X95819, respectively) (12).

\subsection{Multilocus Sequence Typing Analysis}

Multilocus sequence typing analysis was performed as directed in PubMLST (13). Information regarding the seven structural genes used in the study was confirmed from the MLST website and used for primer designing, followed by PCR (14). Each of the seven structural genes was provided a unique allelic number through PubMLST, and the final ST of A. baumannii was determined using a combination of the unique alleles (Table 1). The correlation between the allelic number of gyrB, a structural gene of $A$. baumannii, and STs was investigated. The relationship between the strains was confirmed by comparing the results of the antimicrobial susceptibility test and a peak analysis (performed using the MALDI-TOF MS).

\subsection{MALDI-TOF MS Analysis}

MALDI-TOF MS analyzed the differences in the protein composition of $A$. baumannii and distinguished between the different groups of Acinetobacter. This method confirmed the spectral peak of a ciprofloxacin-resistant $A$. baumannii strain. A detailed analysis was performed on the spectrum obtained for the A. baumannii target strain in the region between $2,000 \mathrm{~m} / \mathrm{z}$ and $18,000 \mathrm{~m} / \mathrm{z}$ using the SARAMIS database (15). 


\begin{tabular}{|c|c|c|c|}
\hline Locus & & Primer Sequence (5-3) & Amplicon Size (bp) \\
\hline \multirow[t]{3}{*}{ gltA } & & & 722 \\
\hline & Citrato F1: & AATTTACAGTGGCACATTAGGTCCC & \\
\hline & Citrato R12: & GCAGAGATACCAGCAGAGATACACG & \\
\hline \multirow[t]{3}{*}{ gyrB } & & & 594 \\
\hline & gyrB_F: & TGAAGGCGGCTTATCTGAGT & \\
\hline & gyrB_R: & GCTGGGTCTTTTTCCTGACA & \\
\hline \multirow[t]{3}{*}{ gdhB } & & & 774 \\
\hline & GDH SEC F: & ACCACATGCTTTGTTATG & \\
\hline & GDH SEC R: & GTTGGCGTATGTTGTGC & \\
\hline \multirow[t]{3}{*}{ recA } & & & 425 \\
\hline & RA1 & CCTGAATCTTCYGGTAAAAC & \\
\hline & RA2 & GTTTCTGGGCTGCCAAACATTAC & \\
\hline \multirow[t]{3}{*}{ cpn6o } & & & 640 \\
\hline & cpn60_F: & GGTGCTCAACTTGTTCGTGA & \\
\hline & cpn60_R: & CACCGAAACCAGGAGCTTTA & \\
\hline \multirow[t]{3}{*}{ gpi } & & & 456 \\
\hline & gpi_F: & GAAATTTCCGGAGCTCACAA & \\
\hline & gpi_R: & TCAGGAGCAATACCCCACTC & \\
\hline \multirow[t]{3}{*}{ rpoD } & & & 672 \\
\hline & rpoD_F: & ACCCGTGAAGGTGAAATCAG & \\
\hline & rpoD_R: & TTCAGCTGGAGCTTTAGCAAT & \\
\hline
\end{tabular}

Abbreviations: gltA, citrate synthase; gyrB, DNA gyrase subunit B; gdhB, glucose dehydrogenase $B$; recA, homologous recombination factor; cpn60, 60-kDa chaperonin; gpi, glucose-6-phosphate isomerase; $r p o D$, RNA polymerase sigma factor; $\mathrm{F}$, sense primer; $\mathrm{R}$, antisense primer.

\section{Results}

\subsection{Strain Isolation}

Of the 98 strains of A. baumannii isolated from clinical specimens, 64 were eventually selected through biochemical testing using VITEK® 2 XL (bioMérieux) and PCR detec-

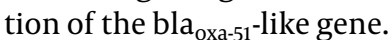

\subsection{Antimicrobial Susceptibility Test}

The resistance rates of $A$. baumannii were $78.1 \%(n=50)$ for imipenem and meropenem, $100 \%(n=64)$ for aztreonam, 79.7\% $(n=51)$ for cefotaxime, $73.4 \%(n=47)$ for ceftazidime, $79.7 \%(n=51)$ for cefepime, $45.3 \%(n=29)$ for aminoglycosides, and 59.3\% $(n=38)$ for amikacin and gentamicin. Among the quinolones tested, a resistance rate of $78.1 \%(n=50)$ was observed for ciprofloxacin. Meanwhile, multidrug-resistant A. baumannii (MRAB), which is simultaneously resistant to at least three antibiotic classes (including $\beta$-lactams, carbapenems, aminoglycosides, and quinolones), showed $57.8 \%$ resistance $(n=37)$.

\subsection{Efflux Pump Inhibition Test}

Fifty ciprofloxacin-resistant strains (MIC $\geq 4 \mu \mathrm{g} / \mathrm{mL}$, as indicated in the antimicrobial susceptibility test) were evaluated in the efflux pump inhibition test using $\mathrm{Pa} \beta \mathrm{N}$ (Appendix 1 in Supplementary File). The MIC of ciprofloxacin for 18 strains was lower in presence of $\mathrm{Pa} \beta \mathrm{N}$ than that in its absence, and the reduction in MIC was four-fold for 2 strains and two-fold for 16 strains. The MIC of levofloxacin reduced for 43 strains. The reduction was eight-fold for 2 strains, four-fold for 18 strains, and two-fold for 23 strains.

\subsection{Sequence Analysis of $Q R D R$ in gyrA and parC}

In all 50 strains of $A$. baumannii, the codon for serine (TCA) at the 83rd position in the gyrA sequence was replaced by that for leucine (TTA) by a point mutation. In 43 strains, the codon for serine (TCG) at the 80th position in parC sequence was replaced by that for leucine (TTG); this mutation was not observed in 7 strains. Therefore, mutations were identified in both gyrA and parC in 43 strains (Figure. 1).

\subsection{Multilocus Sequence Typing Analysis}

Multilocus sequence typing analysis of 64 A. baumannii strains showed 18 STs and allelic numbers that determined the STs (Table 2). The most frequently detected allele expressed in five types of STs, including ST191, and was gyrB3 (62.5\%; n = 40). ST191, ST229, ST369, ST451, ST784, ST1223, ST1225, ST1249, and ST1329 showed resistance to ciprofloxacin, whereas ST229, ST369, ST451, ST784, ST1223, ST1225, ST1249, and ST1329 showed resistance to imipenem, meropenem, gentamicin, and amikacin. ST191 showed resistance to ciprofloxacin, imipenem, meropenem, amikacin, and gentamicin in 21 strains and intermediate resistance in 1 strain (Table 3 ).

\subsection{MALDI-TOF MS Analysis}

Several specific peaks ranging from 2,586 - 17,024 m/z were identified in the spectral analysis. A peak was observed at $5,747 \mathrm{~m} / \mathrm{z}$ for 54 target strains, which is characteristic of A. baumannii. A peak at $9,320 \mathrm{~m} / \mathrm{z}$ was observed for 52 strains, followed by peaks at 7,434 m/z in $68.8 \%(\mathrm{n}=44)$, $4,158 \mathrm{~m} / \mathrm{z}$ in $62.5 \%(\mathrm{n}=40)$, and $12,117 \mathrm{~m} / \mathrm{z}$ in $48.4 \%(\mathrm{n}=31)$ of the strains. Classification based on resistance to major antibiotics using the obtained peak, $78 \%(n=50)$ of the strains with ciprofloxacin resistance showed peaks at 2,586 -2,827 $\mathrm{m} / \mathrm{z}, 6,091-7,273 \mathrm{~m} / \mathrm{z}, 7,464 \mathrm{~m} / \mathrm{z}, 9,092 \mathrm{~m} / \mathrm{z}$, and 11,285 m/z. The results were similar to those obtained for imipenem and meropenem, as $78 \%(n=50)$ strains showed resistance to these antibiotics. 


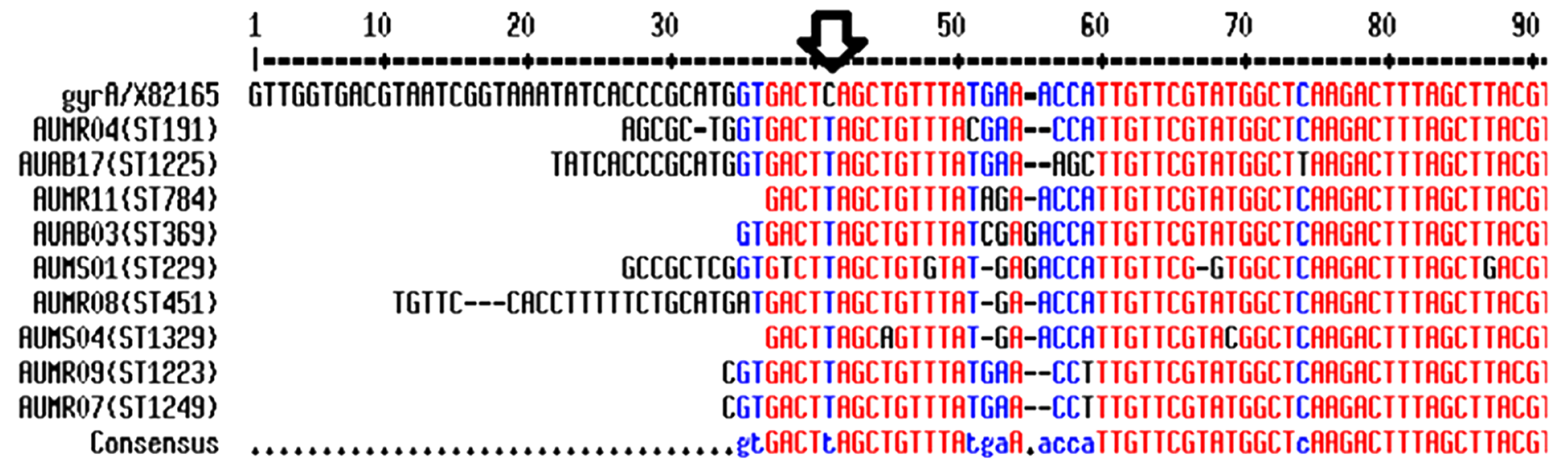

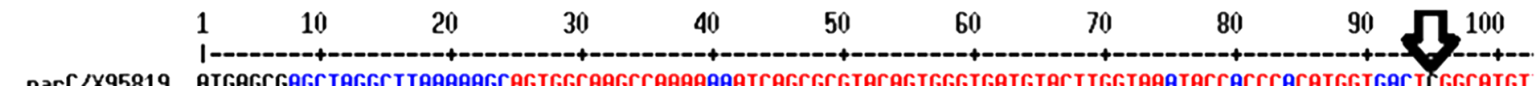

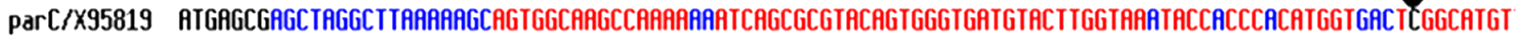

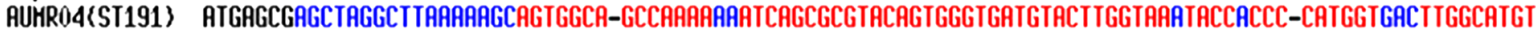
ATGAGCGAGCTCGGCTTATARAGGCAGTGGCAAGCCACAAAAATTCAGCGCGTACAGTGGGTGATGTACTTGGTAA-TACCACCA-CATGGTGACTTGGCATGT GAGCGAGCTAGGCTTAAAAATCAGTGGCAAGCCAAPAATATCAGCGCGTACAGTGGGTGATGTACTTGGTAAR-ACCACCCACATGGTGACTTGGCATGT'

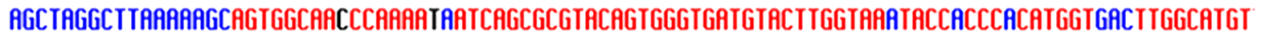
GAGCGAGCTAGGCTIA-AAAGCAGTGGCAAGCCARA月--ATCAGCGCGTACAGTGGGTGATGTACTTGGTAAATACCACCCACATGGTIA-ITGGCATGT

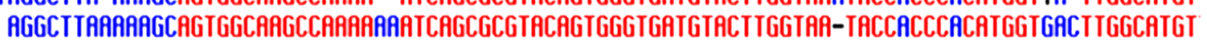

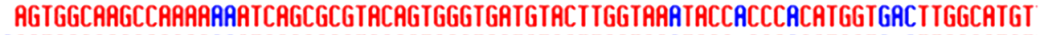
AAAAGCAGTGGCAAGCCAAAAAAATCAGCGCGTACAGTGGGTGATGTACTTGGTAAATACC-CCCACATGGTG-CTTGGCATGT

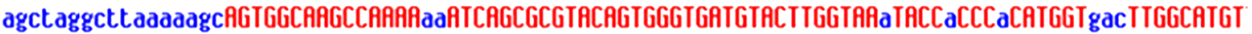

Figure 1. Mutation site marked with an arrow in the two images. Comparison of the mutations in gyrA and parC in Acinetobacter baumannii, with sequencing of wild-type gyrA and parC genes (GenBank Accession No. X82165 and X95819, respectively).

\section{Discussion}

Acinetobacter baumannii is an opportunistic infectious bacterium that causes severe nosocomial infections. The emergence of MDR strains has also been a frequent occurrence in recent years (16). Kumburu et al. (17) reported a result slightly different from this study, with $50 \%$ ciprofloxacin resistance in MRAB strain causing infection associated with wounds and pus, which was isolated from hospitals and clinics in Tanzania (Africa) from 2013 - 2015. However, the resistance rates reported for ceftazidime $(78.6 \%, \mathrm{n}=11)$ and gentamicin $(64.3 \%, \mathrm{n}=9)$ were similar to those obtained in this study. Sequence analysis was performed to identify potential mutations in the gyrA and parC sequences of the 50 ciprofloxacin-resistant $A$. baumannii strains.

In gyrA, the codon for serine at the 83rd position was replaced by that for leucine via a point mutation in all the 50 A. baumannii strains. In $86 \%(n=43)$ of the strains, the codon for serine at the 80th position of parC was replaced by the codon for leucine. Lee et al. (18) reported a new point mutation with tryptophan at the Ser-80 position in parC. Thus, high ciprofloxacin resistance was confirmed when the point mutations inducing the replacement of Ser-83 by Leu and Ser-80 by Trp occurred simultaneously. Here, the 43 strains with both gyrA and parC mutations had relatively high MIC values $(32-256 \mu \mathrm{g} / \mathrm{mL})$ compared to that of strains with only gyrA mutations.

Meanwhile, MLST analysis of the 64 strains showed the presence of 18 STs, and ST191 accounted for the majority $(39 \%, \mathrm{n}=25)$ (Table 2$)$. In a study on the gene cassette and molecular typing of $A$. baumannii integrons isolated from clinical specimens (19), ST195, ST208, ST218, and ST368 were isolated from regions in the vicinity of Shaanxi and Xi'an, China. The STs belonging to CC92 were slightly different from those obtained previously (ST208 and ST1145), suggesting that CC92 exhibited global and regional differences not only in European countries such as Italy, Spain, United Kingdom, Greece, and Denmark, but also in Asian countries such as China, Korea, Thailand, India, and Lebanon. CC92 produces various carbapenemases and is considered the largest and most widely distributed clone worldwide, accounting for the majority of MRAB strains (19). In addition, AUAB08, AUMR02, and AUMR22, identified under ST191, had mutations in both gyrA and parC, and the MICs were as high as $256 \mu \mathrm{g} / \mathrm{mL}$. In contrast, AUAB22, AUMR17, and AUMR26, also identified under ST191, had mutations only in gyrA, and the MICs were relatively low (32$64 \mu \mathrm{g} / \mathrm{mL}$ ). This suggests that even within the same ST, differences in sensitivity may occur due to the presence of var- 


\begin{tabular}{|c|c|c|c|c|c|c|c|c|}
\hline \multirow{2}{*}{ ST } & \multirow{2}{*}{ Number of Isolates (\%) } & \multicolumn{7}{|c|}{ Allelic Number } \\
\hline & & gltA & gyrB & $\operatorname{gdhB}$ & recA & cpn6o & gpi & rpoD \\
\hline ST191 & $25(39.0)$ & 1 & 3 & 3 & 2 & 2 & 94 & 3 \\
\hline ST195 & $2(3.1)$ & 1 & 3 & 3 & 2 & 2 & 96 & 3 \\
\hline ST229 & $6(9.3)$ & 1 & 15 & 2 & 28 & 1 & 107 & 32 \\
\hline ST369 & $3(4.6)$ & 1 & 3 & 3 & 2 & 2 & 106 & 3 \\
\hline ST451 & $5(7.8)$ & 1 & 3 & 3 & 2 & 2 & 142 & 3 \\
\hline ST552 & $2(3.1)$ & 21 & 35 & 2 & 28 & 1 & 145 & 4 \\
\hline ST595 & $1(1.5)$ & 1 & 15 & 66 & 12 & 33 & 90 & 41 \\
\hline ST784 & $5(7.8)$ & 1 & 3 & 3 & 2 & 2 & 107 & 3 \\
\hline ST836 & $1(1.5)$ & 18 & 48 & 58 & 42 & 4 & 54 & 26 \\
\hline ST852 & $1(1.5)$ & 33 & 12 & 40 & 26 & 32 & 157 & 5 \\
\hline ST955 & $1(1.5)$ & 21 & 15 & 2 & 28 & 1 & 157 & 4 \\
\hline ST1181 & $1(1.5)$ & 33 & 31 & 2 & 28 & 1 & 144 & 5 \\
\hline ST1223 & $1(1.5)$ & 10 & 53 & 4 & 64 & 4 & 98 & 5 \\
\hline ST1225 & $3(4.6)$ & 1 & 15 & 13 & 60 & 4 & 106 & 2 \\
\hline ST1249 & $1(1.5)$ & 1 & 15 & 13 & 60 & 4 & 163 & 2 \\
\hline ST1329 & $1(1.5)$ & 1 & 1 & 13 & 12 & 94 & 144 & 2 \\
\hline ST1994 & $1(1.5)$ & 56 & 104 & 137 & 98 & 51 & 191 & 74 \\
\hline ST2114 & $2(3.1)$ & 1 & 12 & 12 & 64 & 4 & 103 & 3 \\
\hline ND & $1(1.5)$ & 1 & 1 & 66 & 60 & 33 & 232 & 41 \\
\hline ND & $1(1.5)$ & 32 & 141 & 81 & 43 & 108 & 149 & 45 \\
\hline
\end{tabular}

Abbreviations: ST, sequence type; ND, sequence type could not be determined.

\begin{tabular}{|c|c|c|c|c|}
\hline \multirow{2}{*}{ ST } & \multicolumn{4}{|c|}{ Antibacterial Susceptibility } \\
\hline & CIP & $\operatorname{IMP} / \operatorname{MEP}(\mathbf{n})$ & $\operatorname{AMK}(\mathbf{n})$ & $\operatorname{GEN}(\mathbf{n})$ \\
\hline ST191 & $\mathrm{R}$ & $\mathrm{R}(23)$ & $\mathrm{R}(16)$ & $\mathrm{R}(21), \mathrm{I}(1)$ \\
\hline ST229 & $\mathrm{R}$ & $\mathrm{R}$ & $\mathrm{R}(1)$ & $\mathrm{R}(1)$ \\
\hline ST369 & $\mathrm{R}$ & $\mathrm{R}$ & $\mathrm{R}$ & $\mathrm{R}$ \\
\hline ST451 & $\mathrm{R}$ & $\mathrm{R}$ & $\mathrm{R}(2)$ & $\mathrm{R}(4)$ \\
\hline ST784 & $\mathrm{R}$ & $\mathrm{R}$ & $\mathrm{R}(4)$ & $\mathrm{R}$ \\
\hline ST1223 & $\mathrm{R}$ & $\mathrm{R}$ & $\mathrm{R}$ & $\mathrm{R}$ \\
\hline ST1225 & $\mathrm{R}$ & $\mathrm{R}$ & $S$ & $S$ \\
\hline ST1249 & $\mathrm{R}$ & $\mathrm{R}$ & $\mathrm{R}$ & $\mathrm{R}$ \\
\hline ST1329 & $\mathrm{R}$ & $S$ & $S$ & $S$ \\
\hline
\end{tabular}

ious ciprofloxacin resistance mechanisms.

Meanwhile, the results of MALDI-TOF MS analysis revealed six common peaks $(4158,4264,5747,7434,8487$, and $9320 \mathrm{~m} / \mathrm{z}$ ) in spectra of most of the strains, and characteristic peak of $A$. baumannii at $5,747 \mathrm{~m} / \mathrm{z}$ was observed in

spectra for all 64 target strains. The major peaks appeared at $9,320,7,434$, and $12,117 \mathrm{~m} / \mathrm{z}$, which is in contrast to the previous reports where the main peaks of $A$. baumannii formed at $4,244,8,485$, and $5,747 \mathrm{~m} / \mathrm{z}(20)$. Upon classifying the strains (exhibiting different antibiotic resistance patterns) according to their peaks, the spectra of $78 \%$ (n $=50$ ) of strains resistant to ciprofloxacin, imipenem, and meropenem were observed to display relatively high intensity peaks at 2,586 - 2,827 m/z, 6,091- 7,273 m/z, 7,464 $\mathrm{m} / \mathrm{z}, 9,092 \mathrm{~m} / \mathrm{z}$, and $11,285 \mathrm{~m} / \mathrm{z}$, whereas this pattern was observed in only $19 \%(n=12)$ of the susceptible strains. In a previous study (21), MALDI-TOF MS and antibiotic resistance analysis for A. baumannii showed that the positions of high-intensity peaks vary with the antibiotic resistance patterns. Here, a peak was detected at $2,033 \mathrm{~m} / \mathrm{z}$ in the spectra of 213 colistin-resistant $A$. baumannii strain isolated from clinical settings.

\subsection{Conclusions}

Here, most of the A. baumannii strains exhibited multidrug resistance to various antibiotics, including ciprofloxacin and levofloxacin. Our findings will be useful 
in antibiotic selection during the treatment of infections caused by MRAB. These findings may also help to determine the effective antibiotic concentration and the methods for preventing group infection and its spread.

\section{Supplementary Material}

Supplementary material(s) is available here [To read supplementary materials, please refer to the journal website and open PDF/HTML].

\section{Footnotes}

Authors' Contribution: C.H., \& J.K. contributed equally to this work. Study concept and design: C. H., J. K., and G. K.; Acquisition of data: C. H., and J. K.; Analysis and interpretation of data: C.H., J. K., and G. K.; Drafting of the manuscript, Critical revision of the manuscript for important intellectual content, Statistical analysis: C. H., and J. K.; Administrative, technical, and material support, Study supervision: G. K.

Conflict of Interests: There was no conflict of interest.

Ethical Approval: This study was approved by the Institutional Review Board (IRB-202010027) of Dankook University.

Funding/Support: There was no funding/support.

\section{References}

1. Hua X, Shu J, Ruan Z, Yu Y, Feng Y. Multiplication of blaOXA-23 is common in clinical Acinetobacter baumannii, but does not enhance carbapenem resistance. J Antimicrob Chemother. 2016;71(12):3381-5. doi: 10.1093/jac/dkw310. [PubMed: 27494931].

2. Seifert H, Muller C, Stefanik D, Higgins PG, Miller A, Kresken $\mathrm{M}$. In vitro activity of sulbactam/durlobactam against global isolates of carbapenem-resistant Acinetobacter baumannii.J Antimicrob Chemother. 2020;75(9):2616-21. doi: 10.1093/jac/dkaa208. [PubMed: 32516359].

3. Mogle BT, Steele JM, Thomas SJ, Bohan KH, Kufel WD. Clinical review of delafloxacin: a novel anionic fluoroquinolone. J Antimicrob Chemother. 2018;73(6):1439-51. doi: 10.1093/jac/dkx543. [PubMed: 29425340].

4. Webber MA, Piddock LJ. The importance of efflux pumps in bacterial antibiotic resistance. J Antimicrob Chemother. 2003;51(1):9-11. doi: 10.1093/jac/dkg050. [PubMed: 12493781].

5. Estrada AA, Gottschalk M, Rossow S, Rendahl A, Gebhart C, Marthaler DG. Serotype and genotype (multilocus sequence type) of streptococcus suis isolates from the united states serve as predictors of pathotype. J Clin Microbiol. 2019;57(9). doi: 10.1128/JCM.00377-19. [PubMed: 31243086]. [PubMed Central: PMC6711919].

6. Teixeira AB, Barin J, Hermes DM, Barth AL, Martins AF. PCR assay based on the gyrB gene for rapid identification of acinetobacter baumannii-calcoaceticus complex at specie level. J Clin Lab Anal. 2017;31(3). doi: 10.1002/jcla.22046. [PubMed: 27605498]. [PubMed Central: PMC6816819].
7. Ghebremedhin M, Heitkamp R, Yesupriya S, Clay B, Crane NJ. Accurate and rapid differentiation of acinetobacter baumannii strains by raman spectroscopy: A comparative study. J Clin Microbiol. 2017;55(8):2480-90. doi: 10.1128/JCM.01744-16. [PubMed: 28592553]. [PubMed Central: PMC5527427].

8. Uddin F, McHugh TD, Roulston K, Platt G, Khan TA, Sohail M. Detection of carbapenemases, AmpC and ESBL genes in Acinetobacter isolates from ICUs by DNA microarray. J Microbiol Methods. 2018;155:19-23. doi: 10.1016/j.mimet.2018.11.004. [PubMed: 30423363].

9. Weinstein MP, Campeau S, Sharon CK, Bobenchik AM, Galas MF, Gold $\mathrm{H}$, et al. Performance standards for antimicrobial susceptibility testing m100. Wayne (PA): Clinical and Laboratory Standards Institute; 2020. p. 83-5.

10. Bello-Lopez E, Rocha-Gracia RDC, Castro-Jaimes S, Cevallos MA, VargasCruz M, Verdugo-Yocupicio R, et al. Antibiotic resistance mechanisms in Acinetobacter spp. strains isolated from patients in a paediatric hospital in Mexico. J Glob Antimicrob Resist. 2020;23:120-9. doi: 10.1016/j.jgar.2020.08.014. [PubMed:32916332].

11. Luna BM, Ershova K, Yan J, Ulhaq A, Nielsen TB, Hsieh S, et al. Adjunctive transferrin to reduce the emergence of antibiotic resistance in Gram-negative bacteria. J Antimicrob Chemother. 2019;74(9):26319. doi: 10.1093/jac/dkz225. [PubMed: 31170282]. [PubMed Central: PMC6736376].

12. Blackwell GA, Holt KE, Bentley SD, Hsu LY, Hall RM. Variants of AbGRI3 carrying the armA gene in extensively antibiotic-resistant Acinetobacter baumannii from Singapore. J Antimicrob Chemother. 2017;72(4):1031-9. doi: 10.1093/jac/dkw542. [PubMed: 28073968]. [PubMed Central: PMC5400096].

13. Jolly KA,, Maiden MF. The Public database for molecular typing and microbial genome diversity. 2020, [cited 2020 Jun 17]. Available from: https: //pubmlst.org.

14. Singhal R, Reynolds PR, Marola JL, Epperson LE, Arora J, Sarin R, et al. Sequence analysis of fluoroquinolone resistance-associated genes gyrA and gyrB in clinical mycobacterium tuberculosis isolates from patients suspected of having multidrug-resistant tuberculosis in New Delhi, India. J Clin Microbiol. 2016;54(9):2298-305. doi: 10.1128/JCM.00670-16. [PubMed: 27335153]. [PubMed Central: PMC5005506].

15. Koo SH, Kwon KC, Cho HH, Sung JY. Genetic basis of multidrugresistant Acinetobacter baumannii clinical isolates from three university hospitals in Chungcheong Province, Korea. Korean J Lab Med. 2010;30(5):498-506. doi: 10.3343/kjlm.2010.30.5.498. [PubMed: 20890082].

16. Nordmann P, Poirel L. Emergence of plasmid-mediated resistance to quinolones in Enterobacteriaceae. J Antimicrob Chemother. 2005;56(3):463-9. doi: 10.1093/jac/dki245. [PubMed: 16020539].

17. Kumburu HH, Sonda T, van Zwetselaar M, Leekitcharoenphon P, Lukjancenko O, Mmbaga BT, et al. Using WGS to identify antibiotic resistance genes and predict antimicrobial resistance phenotypes in MDR Acinetobacter baumannii in Tanzania. J Antimicrob Chemother. 2019;74(6):1484-93. doi: 10.1093/jac/dkz055. [PubMed: 30843063]. [PubMed Central: PMC6524488].

18. Lee JK, Lee YS, Park YK, Kim BS. Mutations in the gyrA and parC genes in ciprofloxacin-resistant clinical isolates of Acinetobacter baumannii in Korea. Microbiol Immunol. 2005;49(7):647-53. doi: 10.1111/j.13480421.2005.tb03643.x. [PubMed:16034208].

19. Temgoua FT, Wu L. Mechanisms efflux pumps of $<\mathrm{i}>$ acinetobacter baumannii</i> (MDR): Increasing resistance to antibiotics. J Biosci Med. 2019;7(1):48-70. doi: 10.4236/jbm.2019.71006.

20. Mari-Almirall M, Cosgaya C, Higgins PG, Van Assche A, Telli M, Huys G, et al. MALDI-TOF/MS identification of species from the Acinetobacter baumannii (Ab) group revisited: inclusion of the novel A. seifertii and A. dijkshoorniae species. Clin Microbiol Infect. 2017;23(3):210 e1-9. doi: 10.1016/j.cmi.2016.11.020. [PubMed: 27919649].

21. Guler G, Erac B. [Investigation of fluoroquinolone resistance mechanisms in clinical Acinetobacter baumannii isolates]. Mikrobiyol Bul. 2016;50(2):278-86. doi: 10.5578/mb.24126. [PubMed: 27175500]. 